\title{
DEVELOPMENT OF A WEB-BASED WIRELESS TELEMONITORING SYSTEM FOR AGRO-ENVIRONMENT
}

\author{
Keming Du ${ }^{1}$, Zhongfu Sun ${ }^{1, *}$, Huafeng Han ${ }^{1}$, Shuang Liu ${ }^{1}$ \\ ${ }^{1}$ Institute of Environment and Sustainable Development in Agriculture (IEDA), Chinese \\ Academy of Agricultural Sciences (CAAS), No. 12, Zhong-guan-cun South Street, Beijing, \\ 100081, P. R. China \\ * Correspondence author: Zhongfu Sun,Tel/Fax: 86-10-62119558,E-mail: sunzf@263.net
}

Abstract: In order to satisfy the requirements of agro-environmental data acquisition, a project scheme of wireless telemonitoring system was plotted out according to the agricultural characteristics of scattered-sites far from developed community, multiple environmental factors, mutable conditions disturbed by natural disasters, and so forth. Integrated with modern information technology (IT), an environmental data acquisition system, named WITSYMOR V1.0, was developed, which was designed to be compatible with most of common sensors, such as temperature and moisture for air and soil, $\mathrm{CO}_{2}$, air pressure, $\mathrm{PAR}$, total solar radiation, soil $\mathrm{pH}$ and $\mathrm{EC}$, etc. The kernel techniques were to realize a seamless connection between wireless mobile network (GPRS/CDMA) and Internet with TCP/IP software design, the data from the remote agricultural sites were real-timely acquired and transmitted to the central database servers, and could be browsed, applied and downloaded by authorized users at anytime and in anywhere. At present, it has been set up in some agricultural stations, horticultural greenhouses, methane-gas pools, as well as in animal shelters. The results show that the system works stably, and it is much adaptive to monitor various agricultural environmental factors in the sites far away.

Keywords: Data acquisition, Telemonitoring, Sensors, GPRS/CDMA, Agro-environment

Du, K., Sun, Z., Han, H. and Liu, S., 2008, in IFIP International Federation for Information Processing, Volume 259; Computer and Computing Technologies in Agriculture, Vol. 2; Daoliang Li; (Boston: Springer), pp. 799-807. 


\section{INTRODUCTION}

For the requirement of precision management and control technology in modern agriculture, the dependence on production field information has become more and more obvious. With the rapid development of modern science and technology, the application of information technology has become an important mark of agricultural modernization. Application of modern IT began in the late 1970s, in spite of that, its development is quite rapid (Lu et al., 2004). For the recent thirty years, making full use of intelligent information technology to promote agricultural modernization, lots of countries have made great achievements. with support of using such technologies, people could exchange and share information resources rapidly, and acquire convenient services about weather, markets, production, crop planting, and so on, which could make the most of the demand for the market with minimum input and maximum benefit simultaneously.

Controlled environment agriculture has become one of the most representative examples of information technology application in agriculture, which integrates with technologies of sensor, network, automatic control, etc (Cox, 2002). Nevertheless, how to develop a telemonitoring system for agricultural data acquisition is still one of the important problems that should be solved in modern agriculture (Sun, 2005; Wang et al., 2006; McKinion et al, 2004; Sobeih, 2005) for accurate data acquisition of crop growth and environmental factors, timely prediction of crop growth and crop diseases, as well as efficient realization of remote diagnosis and management.

At present, two traditional methods are still prevailing in lots of existing environmental monitoring systems (Sun et al., 2005). One of which is so called onsite closed system that uses a field computer with linkage of a series of sensors. Another one is to keep long-distance communication with leased cable. It is well known that most of monitoring sites in agriculture are far away from communities and administrators, such as typical sites like greenhouses, agricultural and forestry ecosystems, meteorological and hydrological stations, etc, for which it is not easy to get environmental information automatically. By contrary, extra input cost would much increase if the leased cable communication network was adopted. What is more, the position of observed sites changes frequently, and the administrators, who often go out for business from place to place but want to know the information of the observed sites far away from themselves. What should they do? Aimed at all mentioned above, a remote monitoring technical scheme is proposed with seamless connection between wireless communication network and Internet, which could provide a suitable solution for efficiently resolving the problems of remote communication in data acquisition and transmission. It is well-known that the research object is 
in quite accordance with the development trend of current monitoring system for agricultural environment (Messer et al., 2006; Serodio et al., 2001).

\section{STRUCTURAL DESIGN AND KERNEL TECHNOLOGY OF THE SYSTEM}

According to the agricultural characteristics mentioned above, integrating with the former research results (Sun et al., 2006), a structural scheme of wireless telemonitoring system for agricultural environment was optimized, upgraded and validated by practical application. And the wireless telemonitoring system for agricultural environment (WITSYMOR V1.0) was realized. This multi-function modern monitoring system was made combined with environmental factor measuring technology, modern transducer technology, wireless communication technology, and computer network information technology, etc. The GPRS/CDMA wireless communication technology was adopted to resolve the difficult problem of field data transmission field, which is a convenient way to solve the so-called 'final one kilometer bottle-neck problem of information freeway' for information transmission from agricultural field to the users.

In structural design (Figure 1), this system consists of three modules: remote monitoring terminal module in observed site for data acquisition and transmission, database server module for data receiving and saving in central database server, and Web server module for data management and process.

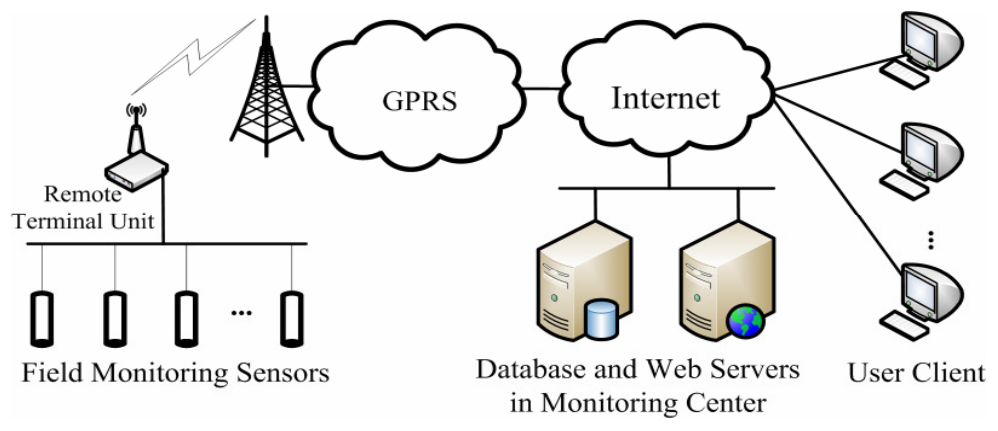

Figure 1. Structure of the system

The following contents are focused on the detailed description about system structure, function, key technology and realization of the three modules. 


\subsection{Remote Monitoring Terminal Module of Data Acquisition and Transmission}

The terminal module is placed in an agricultural observed site, which is responsible for achieving real-time data and sending them to remote central database server through the seamless connection between GPRS/CDMA wireless communication network and Internet.

The hardware device of the module consists of programmable RTU (Remote Terminal Unit), sensors for environmental factors, A/D transform device, RS-485 field bus and mobile SIM card. RS-485 acts as a bridge to connect the programmable RTU and the output signal of sensors through A/D transform devices. The A/D module with multi-channel analog signal input could be connected with various types of analog sensors with standard signal output, from $0-5.0 \mathrm{~V}$ voltage or $0-20 \mathrm{~mA}$ electric current. In addition, digital sensors could be directly connected to RTU only through RS-485 cable without A/D transform.

RTU is widely used in the industry for measurement and control. The programmable RTU of the system provides embedded programming interface. Using integrated development environment of Dynamic C, a specific $\mathrm{C}$ language, the program for data acquisition and process, wireless transmission and regulation have been developed. The program execution flow is shown in Figure 2.

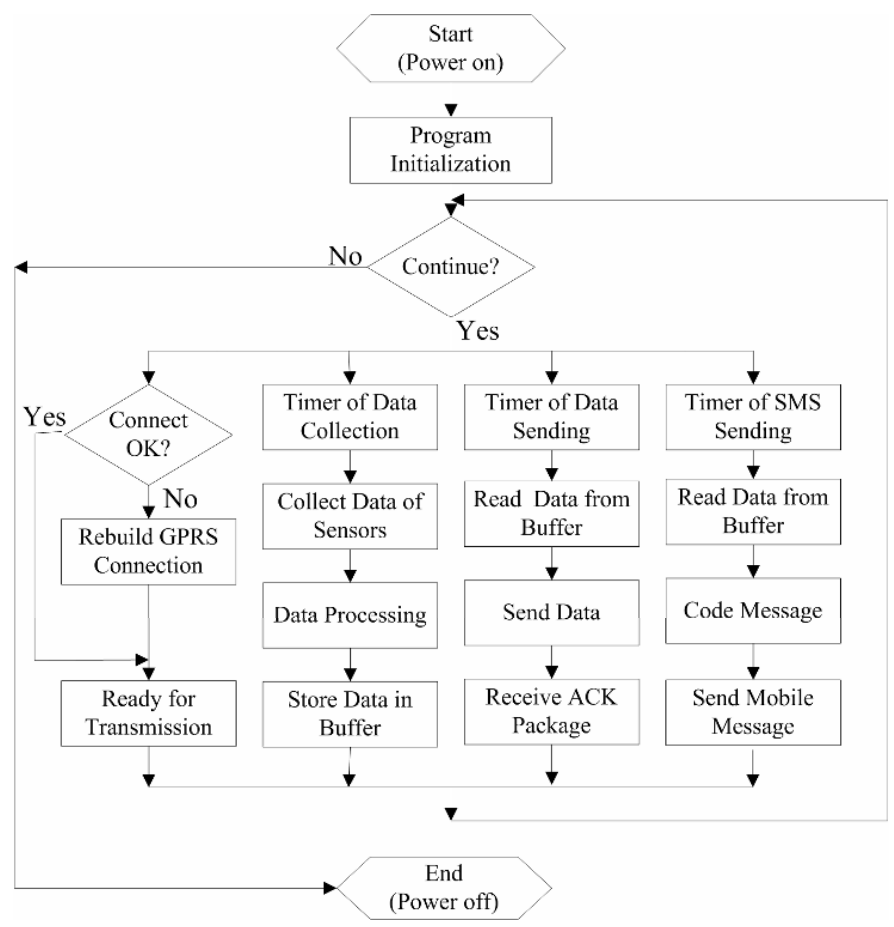

Figure 2. Flow chart of the program for data acquisition and transmission 


\subsection{Central Database Server Module of Data Receiving and Saving}

The database server module acts as a control and management platform consisting of server computer, Microsoft SQL Server database and server application program for data receiving and saving.

DELL PowerEdge 4600 was selected as server computer, and Microsoft SQL Server 2000 as database management. The server application program, installed on the server computer, takes charge of the following functions such as to identify and accept TCP Socket connection request from remote monitoring terminal module, and to analyze and save the data into corresponding database table.

The server application program of the system adopts network programming with Winsock Control Component, using development tool of Microsoft Visual Studio.NET 2003 and program language of VB.NET. The program execution flow is shown in Figure 3.

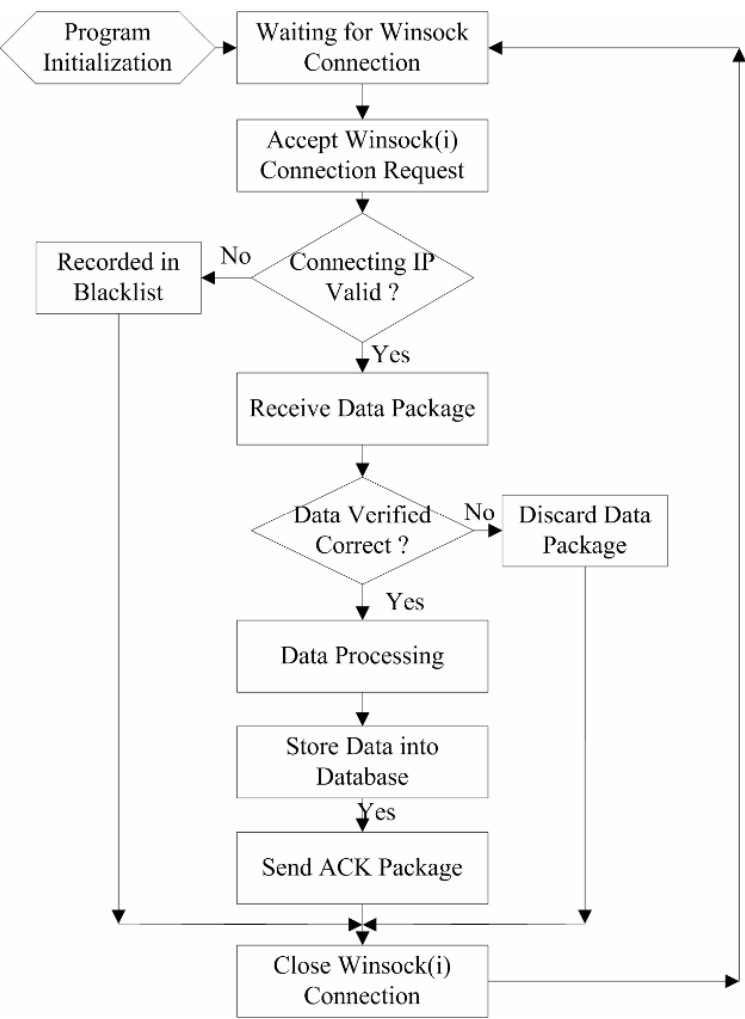

Figure 3. Flow chart of server program for data process 


\subsection{Web Server Module of Data Management}

The Web server module consists of Web server platform and Web server application. The hardware of the Web server adopts enterprise-level server computer (DELL PowerEdge 4600), and for the software of the Web server, Windows 2003 server operation system and IIS6.0 (Internet Information Server) were used. IIS, integrated with Windows operation system, is one of the most popular Web server software, which has advantages of easy configuration and friendly interface.

In order for promoting security and reliability of the database, the system provides a safe scheme that the data could be saved into more than one physical independent servers, which means that the real-time data from the field sites would be sent to two or more different database servers simultaneously.

Authorized users could conveniently access to the following websites e.g. www.sinong.com and www.cea.net.cn, which represent two independent server computer placed in different sites. The Web application of the system adopts B/S (Browser/Server) structure developed with ASP.NET technology, Microsoft Visual Studio.NET 2003 and program language of VB.NET. For the $\mathrm{B} / \mathrm{S}$ structure, the users only need client browser (e.g. IE or Netscape); Most of the processing transaction are executed in Web Server; while processing procedures about data reading, writing and updating are finished in database server, which forms 3-tier B/S structure (Figure 4). Evidently, one of the advantages of this mode is that the client users only need a popular INTERNET Browser, and extra software is not necessary.

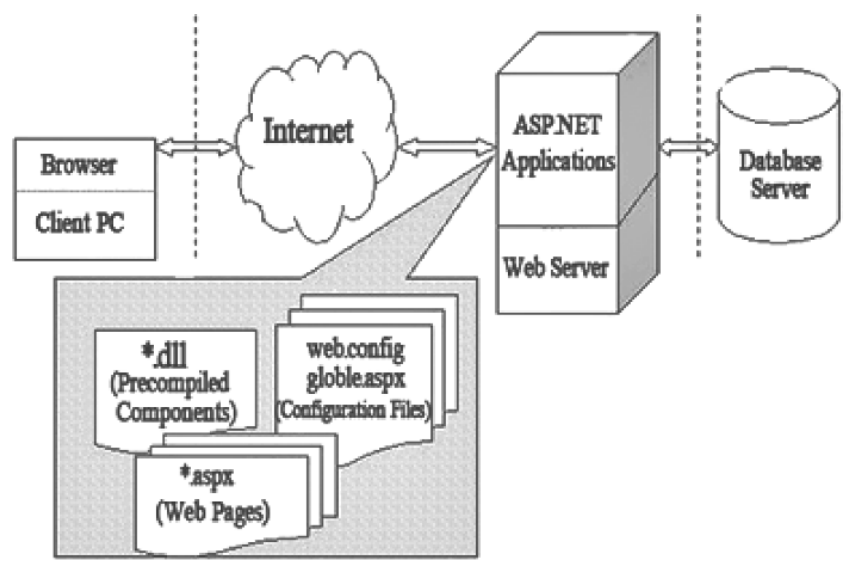

Figure 4. Structure of 3-tier B/S model in the data management module

The execution procedures of the Web server application is as follows: Firstly users have to access to the main Web page for registration through client's browser; Secondly, after achieving authorization, the users could do 
a lot of request operations on the page including real-time data display, historical data inquiries, dynamic data analysis and data file download; And finally, the above requests are submitted to Web server, then the Web server starts to deal with the operations requested and return the results to client's browser. The primary Web pages of data management are shown in Figure 5.

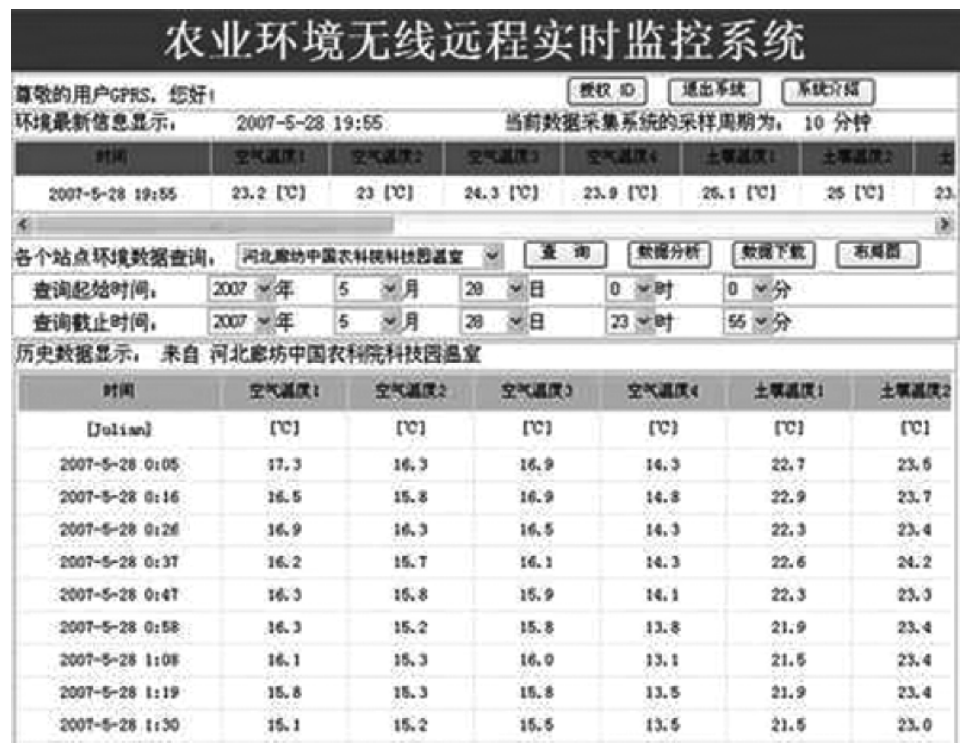

Figure 5. Main page of the system (in Chinese interface)

The Web server application program provides a friendly human-computer interface, by which registered users could browse and analyze the data from remote sites according to a specific object, and similarly, server administrators and production managers also could simultaneously manage information coming from scattered sites at anytime and anywhere.

The most useful functions of the system are summarized as follows:

1) Real-time data display. As soon as the new data arrive to the central server, the Web page refreshes itself automatically.

2) Historical data inquiries. Selecting an observed station, and indicating the starting and ending time, users could make data inquiry of the given period for the selected site.

3) Dynamic analysis. In order to visualize the trend of the data changes with time, the Web server application program provides the function of drawing dynamic curves both for real-time and historical data.

4) Data extraction and download. In order to be convenient for users' download, The Web server provides the function of data transform from MS SQL Server to MS Excel format. 


\section{APPLICATION AND SUMMARY OF THE SYSTEM}

In this paper, an integrated technical solution of the 'field data acquisition - data transmission - Web based data management' was introduced. This system carries out all-weather and real-time data monitoring for various environmental factors. Any user logged in the Web site could use and download the data by accessing to the server. By further analysis of the data, experts could make remote diagnosis and decision support for common farmers.

Up to now, this system has been put into applications in some sites both for practical production and for validation. The observed stations distribute in Liao-ning, He-bei, Guang-dong Provinces, and areas around Beijing, etc. for the data acquisition of greenhouses, methane gas pool and animal farms, the filed scenes of several typical sites equipped with the system are shown in Figure 6. The results show that this system is suitable for the majority of

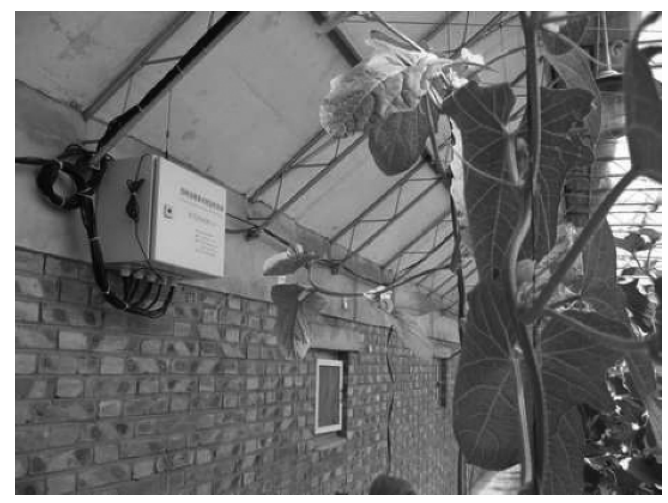

(a) Greenhouse in the garden of CAAS's scientific technology demonstration, Lang-Fang, He-bei Province

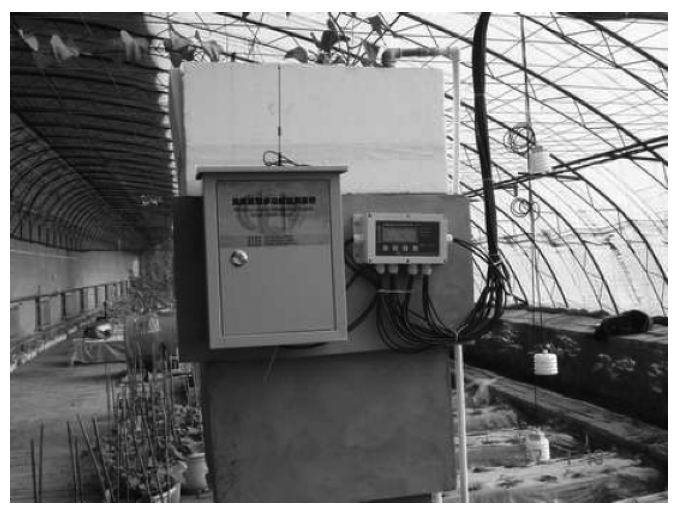

(b) Greenhouse in Daxing District, Beijing, P.R.C

Figure 6. Application scene of the system 
agricultural environment monitoring applications both for research and for practical production.

Although the system has been put into practical applications, and it has been proved to be useful, there still exist some weaknesses to be improved. For example, the system should be further integrated and optimized, the amount of sensors should be expanded to meet more needs, and especially, it is very important to perfect a web-based software that could provide multiple functions, such as data analysis, intelligent control and management for crop cultivation, fertilization and irrigation, as well as for remote pest diagnosis, according to the field data and the combination of expert experiences.

\section{ACKNOWLEDGEMENTS}

This project were jointly supported by National Program-Instrument upgrade and reconstruction (2006JG003500), National Scientific Support Program of Eleventh Five Year Plan (2006BAD04B08), the National Natural Science Foundation of China (30671211)

\section{REFERENCES}

Cox S. Information technology: the global key to precision agriculture and sustainability [J]. Computers and Electronics in Agriculture. 2002, 36: 93-111.

Lu L, Sun J. Agricultural development and modern agricultural construction of China in the new period [J]. Engineering Sciences. 2004, 6(1): 22-29. (in Chinese)

McKinion JM, Willers JL, Jenkins JN. Wireless Local Area Networking for Farm Operations and Farm Management [A]. St. Joseph. ASAE Annual Meeting Papers [C]. Michigan, USA: The American Society of Agriculture Engineers, 2004: 1-16.

Messer H, Zinevich A, Alpert P. Environmental Monitoring by Wireless Communication Networks [J]. Science. 2006, 312: 713-714.

Serodio C, Cunha JB, Morais R, et al. A networked platform for agricultural management systems [J]. Computers and Electronics in Agriculture. 2001, 31: 75-90.

Sobeih A, Chen W, Hou J, et al. J-Sim: A Simulation Environment for Wireless Sensor Networks [A]. Proceedings of the 38th annual Symposium on Simulation [C]. Washington, USA: IEEE Computer Society. 2005: 175-187.

Sun Z, Qiao X, Wang Y. Research and application of IT on controlled environment agriculture. Progress and development in digital agriculture [C]. China Agricultural Science Press, Beijing, 2005: 75-78. (in Chinese)

Sun Z, Cao H, Li H, et al. GPRS and WEB based data acquisition system for greenhouse environment [J]. Transactions of CSAE, 2006, 22(6): 131-134. (in Chinese)

Sun Z, Influence of controlled environment agriculture on modern agriculture of China [J]. Greenhouse \& Horticulture, 2005, (01): 15-17. (in Chinese)

Wang N, Zhang N, Wang M, et al. Wireless sensors in agriculture and food industry-Recent development and future perspective [J]. Computers and Electronics in Agriculture. 2006, 50(1): $1-14$. 